\title{
Her-2 expression in cutaneous eccrine and apocrine neoplasms
}

\author{
Kim M Hiatt, Jessica L Pillow and Bruce R Smoller \\ Department of Pathology, University of Arkansas for Medical Sciences, Little Rock, AR, USA
}

\begin{abstract}
Cutaneous eccrine and apocrine glands have many histologic and immunologic similarities to ducts and acini of the breast. Thus, differentiating a primary cutaneous process from a metastatic breast carcinoma can be nearly impossible. In all, $10-34 \%$ of breast carcinomas overexpress HER-2 protein, a membrane-associated protein that functions in cell differentiation, adhesion and motility. As expression of this gene in cutaneous neoplasms has not been well characterized, we sought to determine HER-2 expression in a sample of benign and malignant cutaneous eccrine and apocrine neoplasms and to determine if there is value in using this protein expression in differentiating primary cutaneous from metastatic breast lesions. Totally, 85 primary cutaneous neoplasms and 11 cutaneous metastases from HER-2-positive breast carcinomas were retrieved from archived material at our institute. All cases were evaluated for HER-2 protein expression using the Dako Hercept Test kit. Membranous HER-2 staining was noted in three of the 85 cutaneous adnexal neoplasms: one hidrocystoma and two nodular hidradenomas. Seven of the 11 cutaneous metastases from HER-2-positive breast carcinomas maintained moderate-to-strong HER-2 expression. In conclusion, while $10-34 \%$ of breast carcinomas overexpress the HER-2 protein, only 3.5\% of cutaneous apocrine and eccrine neoplasms in this study stained with the HER-2 antibody. These HER-2-positive cutaneous neoplasms typically do not pose a diagnostic dilemma in the setting of differentiation from breast metastasis. Additionally, although histologically these breast and cutaneous lesions may have morphologic similarities, the relative lack of HER-2 overexpression suggests that they are different nosologically. Finally, this study suggests that HER-2 protein expression can be a useful tool in differentiating a primary cutaneous appendageal neoplasm from HER-2 expressing metastatic breast carcinoma.
\end{abstract}

Modern Pathology (2004) 17, 28-32, advance online publication, 19 November 2003; doi:10.1038/modpathol.3800007

Keywords: HER-2/neu; cutaneous eccrine neoplasms; cutaneous apocrine neoplasm; metastatic breast carcinoma; histochemistry

Eccrine glands, apocrine glands and mammary glands are all derived from ectoderm and form as downgrowths from the epidermis. Thus, it is not surprising that cutaneous apocrine and eccrine neoplasms have striking histologic similarity to ducts and acini of the breast. Accordingly, it can be very difficult to distinguish these primary cutaneous lesions from metastatic breast carcinoma (Figure 1). Differential protein expression of human mammaglobin gene, ${ }^{1}$ estrogen receptor, progesterone receptor and anti-gross cystic disease fluid protein $^{2-5}$ has largely been unsuccessful in differentiating primary cutaneous neoplasms from metastatic breast carcinoma.

The human HER-2 gene (also called $c$-erb-B2 and $n e u$ ) encodes a 1260 amino-acid transmembrane

Correspondence: KM Hiatt, University of Arkansas for Medical Sciences, Department of Pathology, 4301 West Markham St, \#517, Little Rock, AR 72205, USA.

E-mail: HiattKimM@uams.edu

Received 23 June 2003; revised 24 July 2003; accepted 25 August 2003 ; advance online publication, 19 November 2003 protein with tyrosine kinase activity (p185). It is located on chromosome 17 and has significant homology to the human epidermal growth factor receptor, which is located on chromosome 7 . The protein is thought to play a role in cell differentiation, adhesion and motility. ${ }^{6}$ A soluble ligand has not yet been identified; however, the intramembraneous portion of a transmembrane subunit of membrane mucin Muc4 acts as an intramembraneous ligand and activator. ${ }^{7}$

In normal skin, there is prominent HER-2 protein expression in squamous cells, with increased expression in the mid and upper levels; the basal cells stain weakly or not at all. ${ }^{8}$ Moderate expression is seen in squamous cell carcinomas. Basal cell carcinomas have negligible reactivity in the germinative layer and very low-level expression in the more differentiated cells making up the bulk of the tumor. Significant expression is also seen in the secretory cells of the eccrine sweat glands and in the suprabasilar cells of the external root sheath. ${ }^{8}$ 

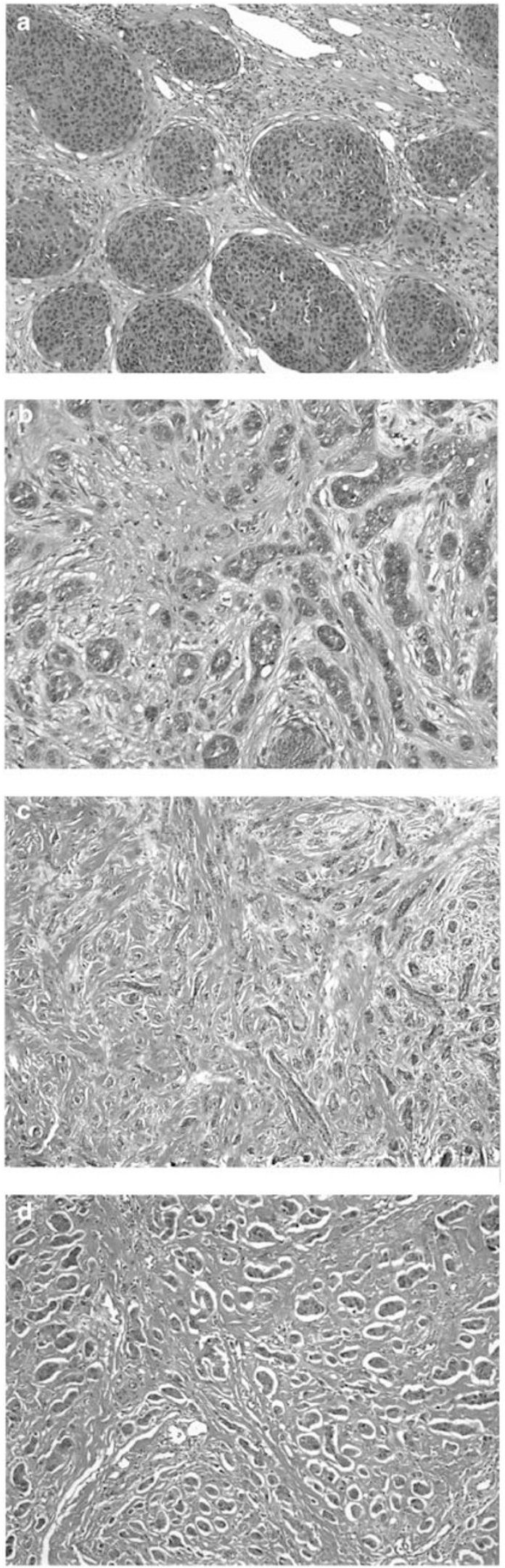

Figure 1 Sections of representative cutaneous lesions: (a) malignant appendageal tumor, (b) eccrine carcinoma, (c) microcystic adnexal carcinoma, and (d) metastatic breast carcinoma.
Overexpression of the HER-2 protein has been reported in $10-34 \%$ of breast carcinomas ${ }^{9}$ and is reported to have prognostic significance. Subsequently, overexpression has been reported in an increasing number of malignancies, including ovarian carcinoma, ${ }^{10}$ osteosarcoma, ${ }^{11}$ invasive bladder carcinoma, ${ }^{12}$ prostate adenocarcinoma, ${ }^{13}$ cholangiocarcinoma, ${ }^{14}$ primary brain tumors ${ }^{15}$ and colon carcinoma, ${ }^{16}$ among others.

HER-2 protein expression in cutaneous neoplasms has not been well characterized. As the distinction between a primary cutaneous adnexal lesion and a metastatic breast carcinoma has significant clinical implications, we sought to determine the expression of the HER-2 protein in primary cutaneous apocrine and eccrine neoplasms and to determine if expression of this protein is a useful tool for distinguishing these lesions from cutaneous metastatic breast carcinoma. The metastatic lesions included in our study were limited to those from primary breast carcinomas with overexpression of the HER-2 protein.

\section{Materials and methods}

Specimens were retrieved from archived material at our institute. All slides were reviewed to confirm the diagnosis. This resulted in 85 primary cutaneous lesions and 11 cutaneous metastases from HER-2 expressing breast carcinomas. Of the primary cutaneous lesions, 14 were malignant, three were classified as atypical (one eccrine poroma and two nodular hidradenomas) and 68 were benign (Table 1).

Metastatic breast lesions were restricted to those with HER-2 overexpression in the primary mammary carcinoma. Of the metastatic breast lesions, the primary carcinoma was recorded as mucinous $(n=1)$, ductal $(n=6)$ or NOS $(n=4)$.

Table 1 Cutaneous lesions included in this study

\begin{tabular}{lc}
\hline Diagnosis & Number of cases \\
\hline Hidrocystoma, eccrine/apocrine & 17 \\
Syringoma & 13 \\
Eccrine poroma & 11 \\
Eccrine spiradenoma & 9 \\
Nodular hidradenoma & 9 \\
Microcystic adnexal carcinoma & 7 \\
Syringocystadenoma papilliferum & 4 \\
Benign appendageal tumor, NOS & 4 \\
Eccrine carcinoma & 3 \\
Eccrine acrospiroma & 2 \\
Mucinous carcinoma & 1 \\
Hidradenoma papilliferum & 1 \\
Eccrine angiomatous hamartoma & 1 \\
Malignant acrospiroma & 1 \\
Aggressive digital papillary carcinoma & 1 \\
Malignant appendageal tumor, NOS & 1
\end{tabular}


30
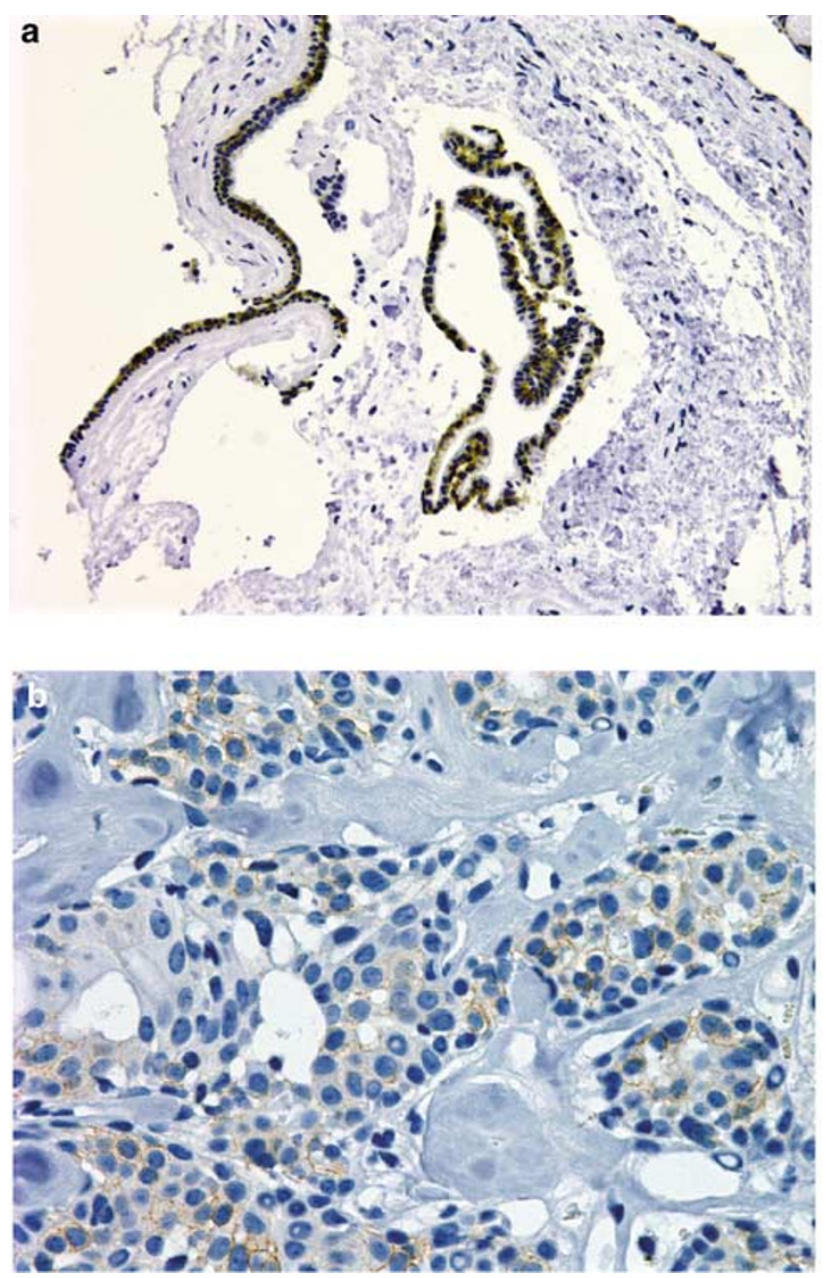

Figure 2 HER-2 staining of primary cutaneous lesions: (a) hidrocystoma and (b) nodular hidradenoma.

HER-2 staining was performed on $4 \mu \mathrm{m}$ sections taken from formalin-fixed, paraffin-embedded tissue. The cut paraffin sections were dried, deparaffinized and rehydrated. Epitope retrieval and automated immunohistochemical staining were performed using the HercepTest kit (DAKO, Carpinteria, CA, USA), an immunohistochemical assay using a goat polyclonal antibody. Any membrane staining with the HER-2 antibody was noted and recorded as diffuse or focal. As the results are not intended for therapeutic purposes, but solely as a diagnostic tool, intensity of staining was not evaluated.

\section{Results}

Three of the 85 primary cutaneous lesions had membraneous HER-2 expression (Figure 2). Two nodular hidradenomas had HER-2 staining, one focally and one diffusely, and one apocrine hidrocystoma had focal membraneous staining. Three additional lesions had cytoplasmic HER-2 staining:
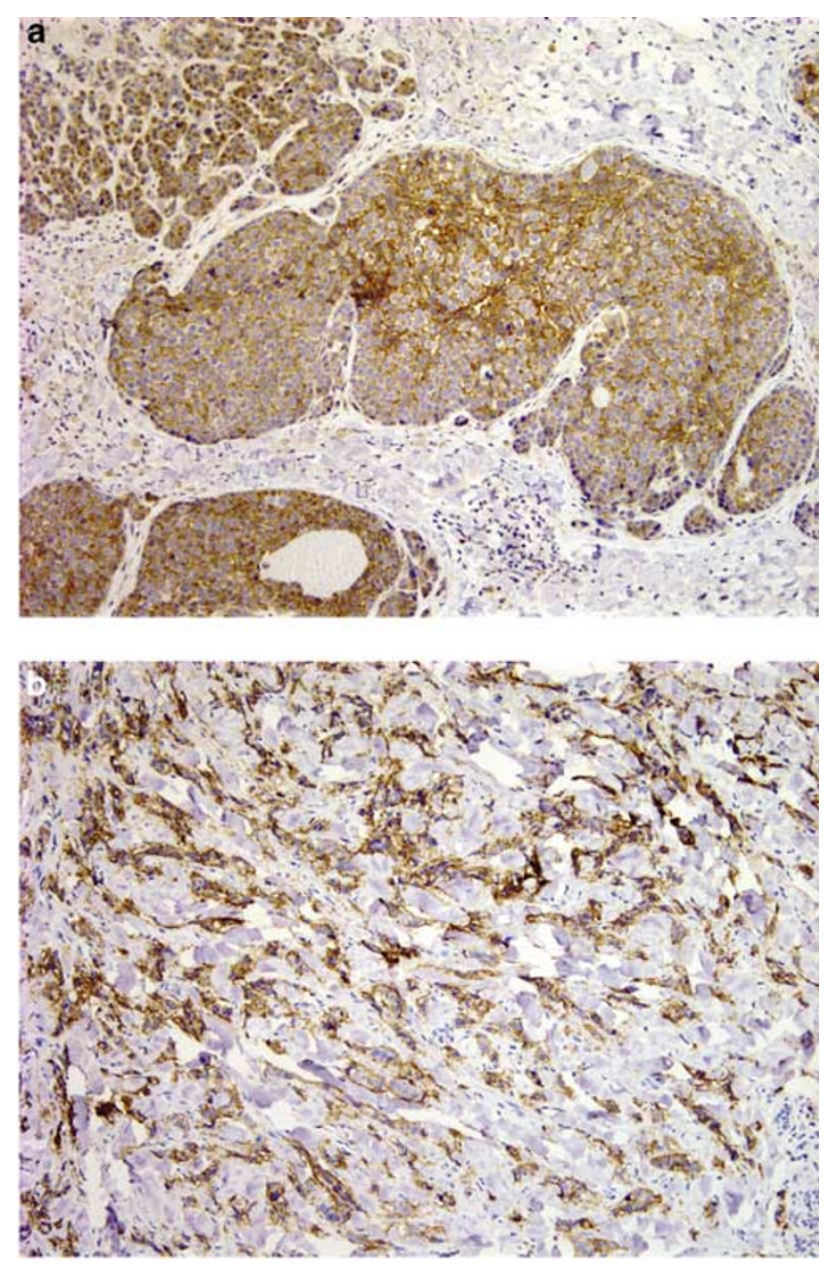

Figure 3 HER-2 staining of cutaneous metastatic breast carcinoma. (a and b)

one hidrocystoma, an eccrine spiradenoma and an aggressive digital papillary adenocarcinoma. As cytoplasmic staining has not been shown to correlate with gene amplification, ${ }^{6}$ only membrane staining was interpreted as significant; therefore, these three lesions with cytoplasmic staining were interpreted as negative.

Seven of the cutaneous metastases from the 11 HER-2-positive breast carcinoma cases retained HER-2 expression (Figure 3). Of the four cases that lost expression, three ductal carcinomas and one classified as NOS, the primary was scored as $3+$ staining in three cases and just recorded as 'positive' in the other case.

\section{Discussion}

Histologic distinction between metastatic breast carcinoma and primary cutaneous adnexal neoplasms on routinely prepared hemotoxylin and eosin stained tissue sections can be very challenging or even impossible. The histological similarities 
among these lesions have been attributed to their common embryologic derivation, in particular their origin as ectodermal downgrowths from the epidermis. Nevertheless, the ability to differentiate these entities has significant clinical implications. Previous studies using antibodies to evaluate the expression of various proteins, including estrogen and progesterone receptors, ${ }^{2-4}$ anti-gross cystic disease fluid protein (BRST-2), ${ }^{2,4}$ carcinoembryonic antigen, ${ }^{4}$ S-100 protein, ${ }^{4}$ and epidermal growth factor, ${ }^{3}$ have shown trends in staining patterns that at best may be helpful, although not entirely diagnostic. There has been no report to date of a single marker, or a panel of markers that reliably makes the distinction among these neoplasms.

Smith et $a l^{17}$ evaluated the staining characteristics of microcystic adnexal carcinoma (MAC), as the prototype for locally aggressive adnexal carcinomas, using a broad panel of immunohistochemical markers, including c-erb-B2. They compared this panel with the staining characteristics of two other primary cutaneous neoplasms, desmoplastic trichoepitheliomas and sclerosing basal cell carcinomas; metastatic breast carcinoma, a great mimic of MAC, was not included. In their study, as in ours, all 10 MACs were negative for c-erb-B2. Another study using primary breast carcinoma and cutaneous ductal sweat gland neoplasms showed overexpression of c-erb-B2 in $33 \%$ of the ductal sweat gland carcinomas and in $20 \%$ of the invasive ductal carcinomas of the breast, ${ }^{4}$ making it an unreliable diagnostic tool in this differential diagnosis. A large study by Busam et $a l^{3}$ evaluated the staining pattern of primary cutaneous sweat gland carcinoma and primary and metastatic breast carcinoma. They showed promising results using antibody against the epidermal growth factor receptor (EGF-R), a protein that has significant homology with HER-2. They showed that $81 \%$ of the sweat gland carcinomas were EGF-R positive, with a predominantly strong, diffuse membraneous pattern. On the other hand, only $17 \%$ of the metastatic breast carcinomas were EGF-R positive, and those tended to have a weak, focal pattern. These results may seem to contradict the findings in this study; however, the genes for these two proteins are located on different chromosomes, and although the proteins have significant homology, they are not the same protein and consequently it is not surprising that they have different expressions. More work is needed to further delineate the functions of these two proteins with striking homology arising from disparate genes.

As evaluation for HER-2 expression is part of the standard work-up for breast carcinoma, we sought to determine if this technique could also be applied for the histologic differentiation of metastatic breast carcinoma from primary cutaneous adnexal neoplasms. Our study broadens the spectrum of cutaneous lesions and compares those with cutaneous metastasis from breast carcinoma with known overexpression of HER-2 in the primary lesion. In all, $77-100 \%$ of HER-2 primary tumors maintain HER-2 expression in metastasis. ${ }^{18,19}$ Our small sampling of 11 cutaneous metastatic tumors, seven $(66 \%)$ of which maintained HER-2 expression, compares favorably with these previous reports. While $10-34 \%$ of breast carcinomas overexpress the HER-2 protein, only three of the $85(3 \%)$ cutaneous apocrine and eccrine neoplasms in this study had any HER-2 expression. Of particular interest is mucinous carcinoma, which in the promising study by Busam et al, ${ }^{3}$ could not be differentiated based on EGF-R positivity; most metastatic breast carcinomas were EGF-R negative as was the primary mucinous sweat gland carcinoma. Our study suggests that HER-2 may be useful in this regard. Our one case of primary cutaneous mucinous carcinomas was negative for HER-2 expression while the metastatic mucinous breast carcinoma retained HER-2 positivity.

Using a rabbit polyclonal antibody directed against a 15-amino-acid sequence of the human neu gene, substantial staining was noted in the secretory cells of the eccrine glands of normal skin. ${ }^{8}$ It is thus surprising that more of the eccrine lesions used in our study did not stain with our HER-2 antibody. This may be explained by the difference in specificity between the rabbit polyclonal antibody directed against a specific polypeptide that was used on normal skin and the goat polyclonal antibody used on our specimens.

Despite similar morphology and embryologic derivations, the lack of EGF-R expression in mammary glands, while being present in most cutaneous neoplasms ${ }^{3}$ in conjunction with the differential staining pattern based on HER-2 expression noted in our study, suggests that cutaneous adnexa and mammary glands are nosologically different from each other.

Nodular hidradenoma and hidrocystoma, the HER-2-positive lesions in this study, do not typically pose a diagnostic dilemma to the practicing pathologist. Thus, in conclusion, although not entirely diagnostic, we propose that HER-2 protein expression can be a useful tool in the panel of markers used in differentiating a primary cutaneous adnexal neoplasm from HER-2 expressing metastatic breast carcinoma.

\section{References}

1 Cole R, Kemp B, Ziesmer S, et al. Detection of Mammaglobin gene (hMAM) expression by mammaglobin 967 in cutaneous metastatic mammary and nonmammary epithelial carcinomas and benign and malignant appendageal tumors [abstract]. American Society of Dermatopathology Annual Meeting 2002.

2 Wallace M, Longacre T, Smoller B. Estrogen and progesterone receptors and anti-gross cystic disease gluid protein 15 (BRST-2) fail to distinguish metastatic 
breast carcinoma from eccrine neoplasms. Mod pathol 1995;8:897-901.

3 Busam KJ, Tan LK, Granter SR, et al. Epidermal growth factor, estrogen, and progesterone receptor expression in primary sweat gland carcinomas and primary and metastatic mammary carcinomas. Mod Pathol 1999;12:786-793.

4 Wick MR, Ockner DM, Mills SE, et al. Homologous carcinomas of the breasts, skin, and salivary glands. A histologic and immunohistochemical comparison of ductal mammary carcinoma, ductal sweat gland carcinoma, and salivary duct carcinoma. Am J Clin Pathol 1998;109:75-84.

5 Ormsby AH, Snow JL, Su WP, et al. Diagnostic immunohistochemistry of cutaneous metastatic breast carcinoma: a statistical analysis of the utility of gross cystic disease fluid protein-15 and estrogen receptor protein. J Am Acad Dermatol 1995;32:711-716.

6 De Potter CR, Schelfhout AM. The neu-protein and breast cancer. Virchows Arch 1995;426:107-115.

7 Carraway KL, Perez A, Idris N, et al. Muc4/sialomucin complex, the intramembrane ErbB2 ligand, in cancer and epithelia: to protect and to survive. Prog Nucleic Acid Res Mol Biol 2002;71:149-185.

8 Maguire Jr HC, Jaworsky C, Cohen JA, et al. Distribution of neu (c-erbB-2) protein in human skin. J Invest Dermatol 1989;92:786-790.

9 Kaptain S, Tan LK, Chen B. Her-2/neu and breast cancer. Diagn Mol Pathol 2001;10:139-152.

10 Gao D, Lu Y, Wang Y, et al. Significance of HER-2/neu expression in ovarian epithelial tumours. Zhonghua Bing Li Xue Za Zhi 2002;31:268-270.

11 Zhou H, Randall R, Brothman A, et al. Her-2/neu expression in osteosarcoma increases risk of lung metastasis and can be associated with gene amplification. J Pediatric Hematol Oncol 2003;25:27-32.

12 Kruger S, Weitsch G, Buttner $\mathrm{H}$, et al. HER2 overexpression in muscle-invasive urothelial carcinoma of the bladder: prognostic implications. Int J Cancer 2002;102:514-518.

13 Sanchez KM, Sweeney CJ, Mass R, et al. Evaluation of HER-2/neu expression in prostatic adenocarcinoma: a request for a standardized, organ specific methodology. Cancer 2002;95:1650-1655.

14 Sirica AE, Lai GH, Endo K, et al. Cyclooxygenase-2 and ERBB-2 in cholangiocarcinoma: potential therapeutic targets. Semin Liver Dis 2002;22:303-313.

15 Forseen S, Potti A, Koka V, et al. Identification and relationship of HER-2/neu overexpression to shortterm mortality in primary malignant brain tumors. Anticancer Res 2002;22:1599-1602.

16 Knosel T, Yr Y, Stein U, et al. Overexpression of cerbB-2 protein correlates with chromosomal gain at the c-erbB-2 locus and patient survival in advanced colorectal carcinomas. Clin Exp Metast 2002;19:401-417.

17 Smith KJ, Williams J, Corbett D, et al. Microcystic adnexal carcinoma: an immunohistochemical study including markers of proliferation and apoptosis. Am J Surg Pathol 2001;25:464-471.

18 Simon R, Nocito A, Hubscher T, et al. Patterns of her-2/ neu amplification and overexpression in primary and metastatic breast cancer. J Natl Cancer Inst 2001;93:1141-1146.

19 Tanner M, Jarvinen P, Isola J. Amplification of HER-2/ neu and topoisomerase II alpha in primary and metastatic breast cancer. Cancer Res 2001;61: 5345-5348. 